\title{
AS MALFORMAÇÕES CONGENITAS NAS INTERNAÇÕES DOS HOSPITAIS DE RIBEIRÃO PRETO, SP, BRASIL
}

\author{
Joaquín Eugenio $\mathrm{Paz} *$ \\ Manildo Fávero** \\ Juan Stuardo Yazlle Rocha ** \\ Nagib Haddad**
}

\begin{abstract}
RSPUB9/424

\begin{tabular}{ll|} 
RSPUB9/424 \\
\hline
\end{tabular}

PAz, J. E. et al. As malformaşōes congênitas nas internações dos hospitais de Ribeirão Preto, SP, Brasil. Rev. Saúde públ., S. Pallo, 12: 356-66, 1978 .

RESUMO: São apresentados os dados sobre internações por malformações congênitas registradas pelo Centro de Processamento de Dados Hospitalares do Departamento de Medicina Social da Faculdade de Medicina de Ribeirão Preto, USP, desde 19 de janeiro de 1970 a 30 de julho de 1974 em sete estabelecimentos hospitalares. Tais internaçöes participam com $1,48 \%$ no total de internações, excluindo-se as causas acidentais. Encontrout-se que as distribuiçóes de diagnósticos principais e secundários classificados por tipo de malformação são significantemente diferentes; descrevell-se a participação de cada estabelecimento nestas internações. Do mesmo modo, encontrou-se um incremento significativo na percentagem de internaçóes por malformaçóes congênitas com o transcurso de tempo, o que é atribuido ao Hospital das Clínicas exclusivamente. Apresentou-se a percentagem de mortalidade por diagnóstico, as razóes de sexo e a duração média das internaçôes, encontrando-se nesta ültima que é maior que a média do total de internações. Descreveu-se também a realizaçấo de procedimentos cirúrgicos, a distribuiçáo por idades e a procedência dos pacientes com malformaçóes congênitas, sugerindo que os hospitais de Ribeirấo Preto funcionem como centros de referência para o tratamento e a correção dessas anomalias.
\end{abstract}

Unitermos: Anomalias congênitas, Ribeirão Preto, SP, Brasil.

\section{N T R O D U A O}

As malformações congênitas constituem um grupo de patologia cuja importância, dentro das enfermidades crônicas comuns, atribui-se tanto à sua freqüência, estimada em $5 \%$ dos nascimentos ${ }^{4}$, como à complexidade de meios que requerem para seu tratamento e correção, à heterogeneidade de formas e etiologia que possuem.
Sabe-se que as malformações congênitas aumentam sua contribuição à mortalidade infantil, à medida que as populações vão progredindo sócio-culturalmente. Assim, por exemplo, na Inglaterra, no período compreendido entre os anos 1951 e 1960 , a mortalidade infantil reduziu-se de 30 a 22 por mil, enquanto que sua proporção relativa

* Do Departamento de Genética do Instituto de Biologia da Universidade Federal do Rio de Janeiro - Caixa Postal 68011 - Ilha do Fundão - 20000 - Rio de Janeiro, RJ - Brasil.

* Do Departamento de Medicina Social da Faculdade de Medicina de Ribeirão Preto da USP - Caixa Postal 301 - 14100 - Ribeirão Preto, SP - Brasil. 
PAZ, J. E. et al. As malformaçōes congênitas nas internações dos hospitais de Ribeirão Preto, SP, Brasil. Rev. Saúde públ., S. Paulo, 12:356-66, 1978.

às malformações congênitas aumentou de 14 a $21 \%$. Ocorre então um descenso marcado na mortalidade por causas infecciosas e carenciais, existindo no entanto, uma tendência estável na freqüência das malformações congênitas ${ }^{6}$. Portanto, deduz-se que é conveniente estudar sua participação relativa nas tarefas dos sistemas de assistência médica e sua evolução com o tempo.

O presente trabalho foi planejado com esse objetivo, para prever as exigências futuras à medida que as condições econômico-sociais forem evoluindo, avaliando a participação das malformações congênitas nas internações registradas pelo Centro de Processamento de Dados Hospitalares (CPDH), do Departamento de Medicina Social da Faculdade de Medicina de Ribeirão Preto da Universidade de São Paulo ${ }^{3}$

\section{MATERIAL E MÉTODOS}

Foram utilizados os dados referentes às internações hospitalares ocorridas até o dia 30 de junho de 1974 e a partir de $1^{\circ}$ de janeiro de 1970, em Ribeirão Preto, no Hospital das Clinicas da Faculdade de Medicina de Ribeirão Preto, Hospital São Francisco, Hospital São Lucas, Hospital dos Acidentados, Instituto Santa Lydia e Santa Casa de Misericórdia; e desde o 1" de abril de 1971 pelo Hospital Imaculada Conceição da Sociedade Portuguesa de Beneficência.

Do total de internaçōes, foram eliminadas aquelas provocadas por acidentes, envenenamento e violência correspondentes ao grupo XVII da Classificação Internacional de Enfermidades, $8^{a}$ Revisão, utilizada na codificação do presente material 5.

Classificou-se como diagnóstico principal aquele que constituiu o motivo da internação, e como diagnósticos secundários aqueles efetuados em pacientes internados por outras causas.

Para os diagnósticos principais de malformações registraram-se os seguintes dados: estabelecimento onde ocorreu a inter- nação, data de internação e alta, sexo, idade e procedência do paciente, ocorrência ou não de cirurgia, e condição de vivo ou morto ao momento da alta.

\section{RESULTADOS E DISCUSSÃO}

\section{Freqüência de diagnóstico de malformações congênitas}

No periodo considerado, num total de 166.792 internaçōes, em 2.475 o diagnóstico principal foi de malformações congênitas, o que equivale a $1,48 \%$ do total. Como diagnóstico secundário, as malformaçōes congênitas foram registradas 964 vezes, ou seja, $0,58 \%$ sobre o total de internações. A Tabela 1 mostra tais cifras classificadas por tipo de malformações congênitas e de diagnóstico.

Considerando a anencefalia e a espinha bifida como uma só categoria, verifica-se que as distribuições de diagnósticos principais e secundários são significativamente diferentes $\left(\mathrm{X}_{18}^{2}=292,62 ; \mathrm{p}<0,001\right)$. Os valores que mais contribuiram para essa diferença correspondem a um excesso nos diagnósticos principais de lábio leporino e/ou palato fendido (749), com uma freqüência relativa de $11 \%$ contra $3 \%$ nos diagnósticos secundários, e de pé torto (754) com $12 \%$ contra $5 \%$ nos secundários. No sentdio inverso, isto é, com menor frequiência relativa de diagnóstico principal, encontram-se as anomalias do coração (746) com $10 \%$ contra $24 \%$ dos diagnósticos secundários, e as malformações múltiplas e sindromes $(759) \quad \mathrm{com} 3 \%$ contra $10 \%$ nos secundários. Estas diferenças podem relacionar-se para sua explicação, com o que ocorre com os diagnósticos principais relativos a cirurgia (Tabela 2). Assim, enquanto o lábio leporino e/ou palato fendido como pé torto registram mais de $90 \%$ de intervençōes cirúrgicas, as anomalias cardíacas $e$ as malformaçōes múltiplas e sindromes são as que com menor freqüência registram tais procedimentos. 
PAZ, J. E. et al. As malformações congênitas nas intcrnaçues dos hospitais de Ribeirão Preto, SP, Brasil. Rev. Saúde públ., S. Paulo, 12:356-66, 1978.

\begin{tabular}{|c|c|c|c|}
\hline \multicolumn{2}{|c|}{$\begin{array}{c}\text { Classificação das internações por malformações congênitas, } \\
=01 / 01 / 1970-30 / 06 / 1974 .\end{array}$} & or diagnóstico & $\mathrm{CPDH}=$ \\
\hline Diagnóstico & $\begin{array}{l}\text { Código } \\
\text { C.I.E. } \\
\text { ne }\end{array}$ & $\begin{array}{l}\text { Diagnóstico } \\
\text { principal } \\
\text { ne }\end{array}$ & $\begin{array}{l}\text { Diagnóstico } \\
\text { secundário } \\
\text { nº }\end{array}$ \\
\hline Anencefalia & 740 & 4 & 1 \\
\hline Espinha Bifida & 741 & 50 & 23 \\
\hline Hidrocefalia Congênita & 742 & 74 & 23 \\
\hline $\begin{array}{l}\text { Outras Anomalias Congênitas do Sistema } \\
\text { Nervoso }\end{array}$ & 743 & 53 & 27 \\
\hline Anomalias Congênitas do Olho & 744 & 70 & 5 \\
\hline $\begin{array}{l}\text { Anomalias Congênitas do Ouvido, da Face e do } \\
\text { Pescoço }\end{array}$ & 745 & 60 & 22 \\
\hline Anomalias Congênitas do Coração & 746 & 242 & 229 \\
\hline Outras Anomalias Congênitas do Aparelho Circulatório & 747 & 69 & 28 \\
\hline Anomalias Congênitas do Aparelho Respiratório & 748 & 17 & 10 \\
\hline Fissura da Abóbada Palatina e Lábio Leporino & 749 & 275 & 33 \\
\hline $\begin{array}{l}\text { Outras Anomalias Congênitas da Parte Superior do } \\
\text { Tubo Digestivo }\end{array}$ & 750 & 52 & 13 \\
\hline Outras Anomalias Congênitas do Aparelho Digestivo & 751 & 101 & 39 \\
\hline Anomalias Congênitas dos órgãos Genitais & 752 & 390 & 166 \\
\hline Anomalias Congênitas do Aparelho Urinário & 753 & 61 & 35 \\
\hline Pé torto (congênito) & 754 & 306 & 47 \\
\hline Outras Anomalias Congênitas dos Membros & 755 & 275 & 85 \\
\hline $\begin{array}{l}\text { Outras Anomalias Congênitas do Sistema ósteo- } \\
\text { Muscular }\end{array}$ & 756 & 223 & 55 \\
\hline Anomalias Congênitas da Pele, do Cabelo e das Unhas & 757 & 29 & 10 \\
\hline Outras Anomalias Congênitas e as não especificadas & 758 & 56 & 19 \\
\hline $\begin{array}{l}\text { Síndromes Congênitas que afetam à Múltiplos Apa- } \\
\text { relhos e Sistemas }\end{array}$ & 759 & 68 & 94 \\
\hline Total & & 2475 & 964 \\
\hline
\end{tabular}

Pode-se estimar, portanto, que enquanto os primeiros são internados fundamentalmente para sua correção, e portanto a malformações congênitas constitui-se em um diagnóstico principal, as segundas são internadas por outras causas que complicam () quadro clinico, passando a malformaçōes congênitas a ser considerada como um diagnóstico secundário.

\section{Participação de cada estabelecimento nas internaçôes}

Na Fig. 1 pode observar-se a participação relativa de cada estabelecimento no total de internaçōes e nas internações com diagnóstico principal de malformações congênitas. É evidente que não são proporcionais: assim, a maior freqüência de internações por malformações congênitas corres- 
PAZ, J. E. et al. As malformações congênitas nas internaçōes dos hospitais de Ribeirão Preto, SP, Brasil. Rev. Saúde públ., S. Paulo, 12:356-66, 1978.

T A B E L 2

Internações com diagnósticos principais de malformaçōes congênitas $=$ razão de sexos, duração média das internações e percentagem delas nas que se realizou algum procedimento cirúrgico. por tipo de malformações congênitas - CPDH $=01 / 01 / 1970-30 / 06 / 1974$.

\begin{tabular}{|c|c|c|c|}
\hline Diagnóstico & $\begin{array}{l}\text { Razão de sexos } \\
(\mathbf{M} / \mathrm{F})\end{array}$ & $\begin{array}{c}\text { Duração Média } \\
\text { da internação } \\
\text { (dias) }\end{array}$ & $\begin{array}{c}\text { Cirurgia } \\
\%\end{array}$ \\
\hline 740 & 0,50 & 48,0 & 0.0 \\
\hline 741 & 0,82 & 31.5 & 73.2 \\
\hline 742 & 1.29 & 26,4 & 61.8 \\
\hline 743 & 0.90 & 20.2 & 39.5 \\
\hline 744 & 2,11 & 23.1 & 73.3 \\
\hline 745 & 0.76 & 7,7 & 86.7 \\
\hline 746 & 1,16 & 19,9 & 21.5 \\
\hline 747 & 1,07 & 17.0 & 60.7 \\
\hline 748 & 0.70 & 20.0 & 58,8 \\
\hline 749 & 1,70 & 9,1 & 97,2 \\
\hline 750 & 1,64 & 31,0 & 86,5 \\
\hline 751 & 1.38 & 15,4 & 81,0 \\
\hline 752 & 4,74 & 7.8 & 86.5 \\
\hline 75.3 & 1.32 & 16.7 & 54.6 \\
\hline 754 & 1,98 & 16.4 & 90,1 \\
\hline 755 & 0.70 & 14,8 & 73.5 \\
\hline 756 & 1,46 & 17.7 & 64,0 \\
\hline 757 & 0,14 & 8.0 & 60,0 \\
\hline 758 & 1,18 & 3,1 & 79,2 \\
\hline 759 & 0,58 & 15,7 & 28,1 \\
\hline Total & 1,45 & 15,2 & 71,8 \\
\hline
\end{tabular}

$\% \quad 40 \quad 30 \quad 20 \quad 10 \quad 0$

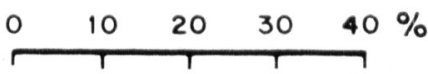

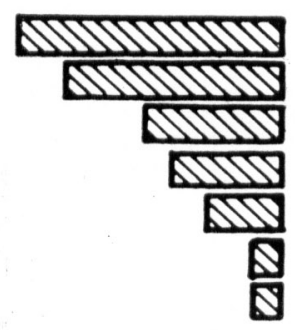

Total

M.C.

Fig. 1 - Participação relativa de cada estabelecimento no total de internações e nas internações por malformações congênitas. CPDH - 01/01/1970 - 30/06/1974. 
PAZ, J. E. et al. As malformações congênitas nas internações dos hospitais de Ribeirão Preto, SP, Brasil. Rev. Saúde púbi., S. Paulo, 12:35ో-66, 1978.

ponde ao Hospital das Clínicas que ocupa o segundo lugar em relação ao total, seguido pelo Instituto Santa Lydia, que com mais de uma quarta parte das internações por malformações congênitas, só participa em $4 \%$ no total de internações. Sendo esta uma instituição especializada em ortopedia explica-se facilmente o fato. enquanto que no caso do Hospital das Clínicas pode contribuir o fato de tratar-se de hospital escola de alta complexidade, unido talvez a outros fatores que serão discutidos mais adiante.

Composição das internações com diagnóstico principal de malformaşöes congênitas

Na Fig. 2 pode observar-se a composição relativa das internaçōes com diagnóstico principal de malformações congênitas para os cinco Hospitais com a maior frequência de tais internações, e para o total dos estabelecimentos. As diferenças podem representar as distintas especialidades praticadas em cada um. Assim, por exemplo, no Instituto Santa Lydia as internações por malformações congênitas ocorrem, em sua grande maioria, por lábio leporino e/ou palato fendido (749) e por pé torto e outras anomalias dos membros (754-755), enquanto que, tanto no Hospital São Francisco como no Hospital São Lucas, a maior frequiência de internações por malformações congênitas referem-se às anomalias dos órgãos genitais (752). As malformações destes órgãos constituem, no total a primeira causa de internações das malformações congênitas
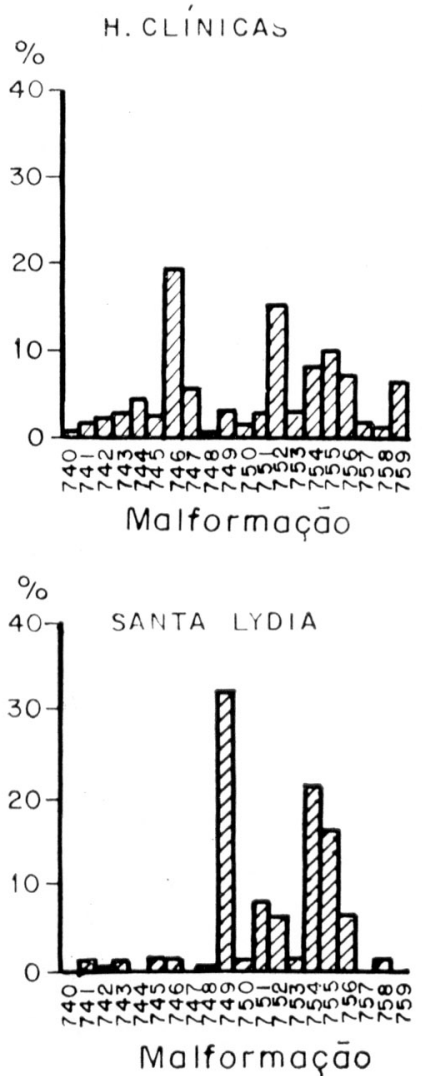

SÄO FRANCISCO
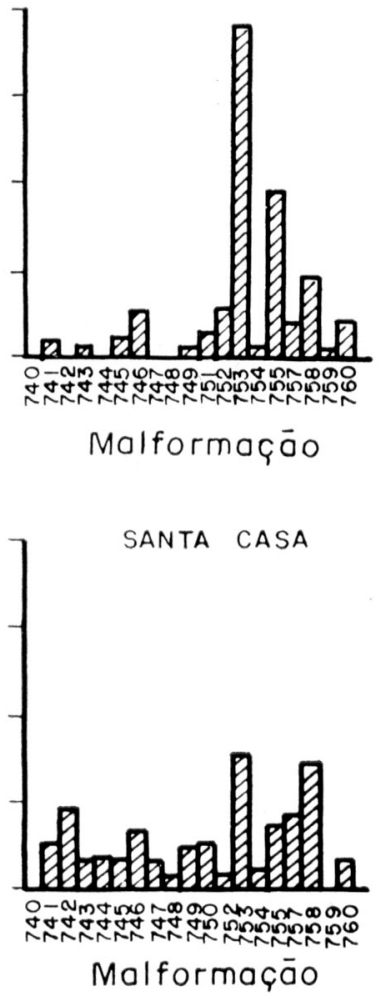

SÁO LUCAS
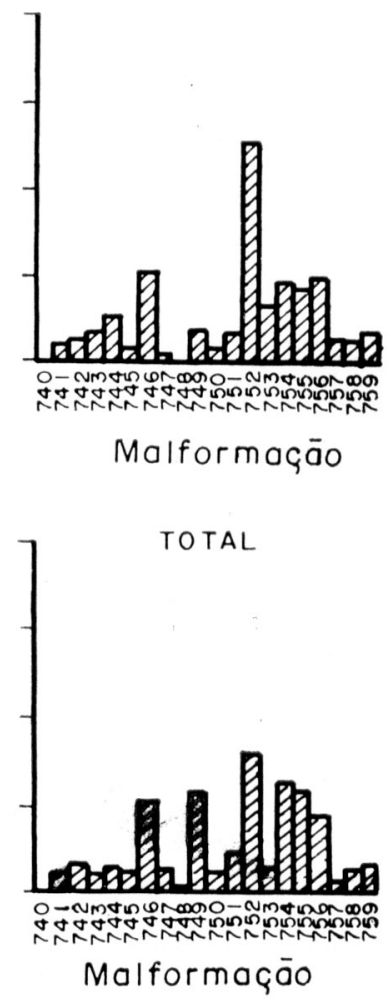

Fig. 2 - Comparação por tipo de malformação nas internações por malformações congênitas para cinco hospitais e para o total. CPDH - 01/01/1970 - 30/06/1974. 
PAZ, J. E. et al. As malformações congênitas nas internações dos hospitais de Ribeirão Preto, SP, Brasil. Rev. Saude públ., S. Paulo, 12:356-66, 1978.

encontrada, embora sejam aquelas que ocupam o sétimo lugar na freqüência relativa no momento do nascimento (Fig. $3)$, sendo precedida pelas malformações múltiplas e síndromes (759), pelas anomalias do ouvido, da face e pescoço (745), por pé torto (754), pelas outras anomalias de membros (755), pelas de pele e fâneros (757) e pelas do aparelho circulatório (747) 2. Apesar de que ambos os grupos (internações e recém-nascidos) não são comparáveis, esta diferença encontrada pode constituir um indicio de que, ou há uma maior preocupação familiar para a correção das malformações genitais, ou então que requeiram mais de uma internação para o seu tratamento, enquanto outras malformações congênitas, como pé torto, são tratadas mais frequientemente nos ambulatórios.

Evolução secular das freqüências de diagnóstico de malformações congênitas nas internações

A Fig. 4 mostra as freqüências de diagnóstico principal e secundário de mal- formações congênitas nas internações classificadas por trimestre e ano de ocorrência. Pode-se observar um aumento significativo nas freqüências de internações com diagnóstico principal de malformações congènitas com o transcurso de tempo $(\mathrm{r}=0,64$; $\mathrm{p}<0,005)$, enquanto que a freqüência de internações com diagnóstico secundário de malformações congênitas permanece ao longo do periodo considerado praticamente estável $(\mathrm{r}=0,05 ; 0,9>\mathrm{p}>0,8)$. Isto poderia refletir um aumento do interesse médico acerca deste tipo de patologia crônica

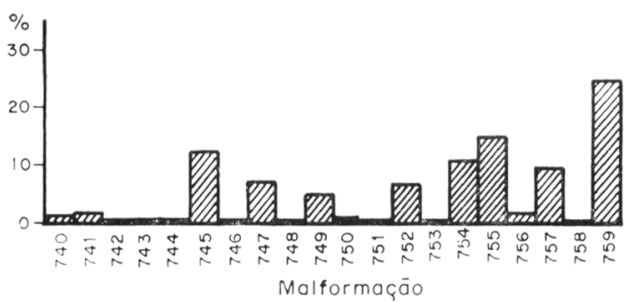

Fig. 3 - Comparação por tipo de malformação das frequiências relativas dos diagnósticos de malformações congênitas efetuados em recémnascidos. ECLAMC 1967-1971.

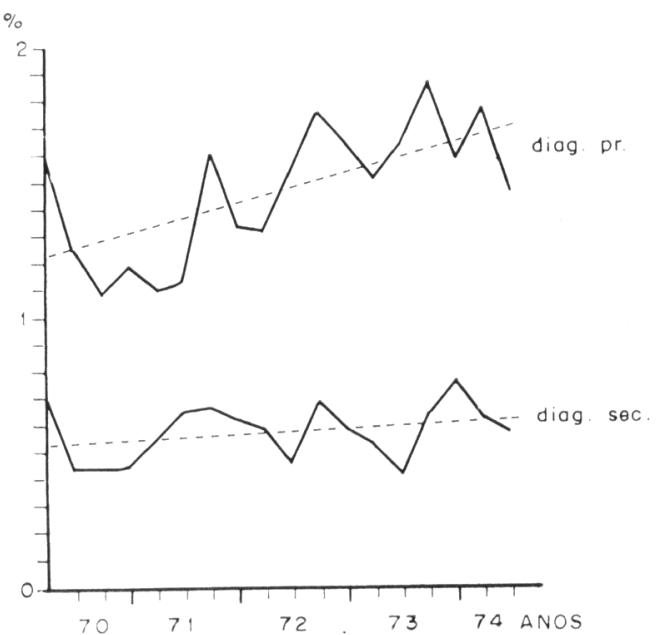

Fig. 4 - Percentagem de intelnações com diagnóstico principal e secundário de malformaçōes congênitas, por trimestre de ocorrência, para todos os estabelecimentos considerados. $\mathrm{CPDH}=01 / 01 / 1970-30 / 06 / 1974$

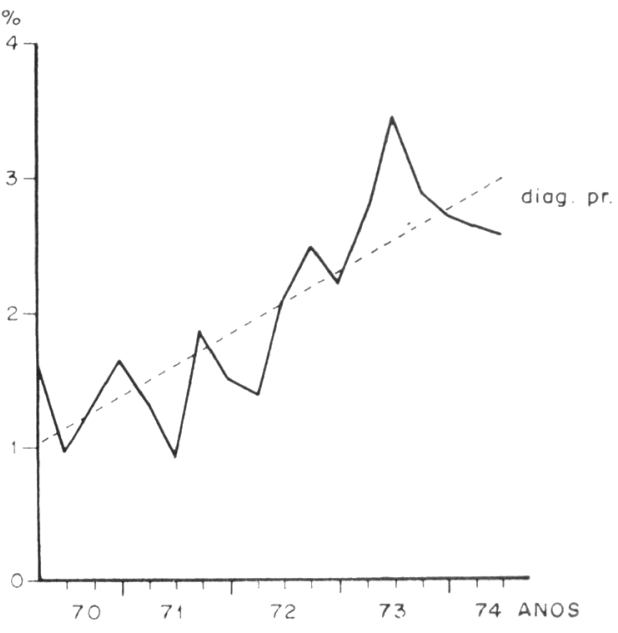

Fig. 4.1. - Percentagem de internações com diagnóstico principal de malformaçōes congênitas, por trimestre de ocorrência, no Hospital das Clínicas da FMRP, CPDH - 01/01/1970 $30 / 06 / 1974$. 
PAZ. J. E. et al. As malformações congênitas nas internações dos hospitais de Ribeirão Preto, SP, Brasil. Rev. Saúde públ., S. Paulo, 12:356-66, 1978.

comum, uma vez que revela que a "necessidade" de assistência médica especializada é de magnitude superior à demanda real. Com a finalidade de testar grosseiramente a primeira hipótese, analisou-se a situação separadamente por estabelecimento, baseando-se no fato seguinte: no Hospital das Clínicas coincide, no período considerado, seu ingresso em um estudo de Castilla e col.2, no ano de 1972, e a instalação de um Consultório de Assessoramento Cienético em 1974; portanto, e devido a isto, deveria haver um incremento na freqüência de internações por malformaçōes congênitas superior ao resto dos estabelecimentos hospitalares, se o interesse médico estivesse envolvido no processo. Verifica-se que o Hospital das Clinicas era o único que apresentava um aumento de frequiência de internações por malformações congênitas no período estudado $(\mathrm{r}=0,83 ; \mathrm{p}<0,001)$ (Fig. 4.1). Vemos também nas Figs. 4 e 4.1 que as internações por malformações congênitas apresentam comportamento ciclico mais ou menos anual, com maior proporção delas no $3^{\circ}$ ou $4^{\circ}$ trimestre de cada ano, no período estudado.
Mortalidade nas internaçóes com diagnóstico de malformaçōes congênitas

Utilizou-se o dado de mortalidade no presente material a partir do primeiro trimestre de 1972. Na Fig. 5 pode-se observar a taxa percentual de mortalidade para as internaçôes com diagnósticos principais e secundários de malformações congênitas. Nos diagnósticos principais a maior mortalidade corresponde às malformações do sistema nervoso central, seguidas por anomalias do tubo digestivo, do aparelho respiratório, das malformações cardíacas, e das múltiplas e síndromes. $O$ restante das malformações congênitas praticamente não está representado na mortalidade, ao contrário do que se sucede nos diagnósticos secundários, evidenciando que freqüentemente as malformações congênitas não são consideradas como causas primeiras de óbitos. Em última instância, isto nāo faz senão refletir a arbitrariedade do conceito de atribuir a uma só causa a morte de um paciente. $O$ coeficiente de mortalidade hospitalar geral, (excluido o grupo XVII) nos anos de 1972 e 1973 foi de $3,4 \%$, enquanto o coeficiente de mortalidade específico para as internações por malformações congênitas foi de $5,1 \%$ no mesmo período.
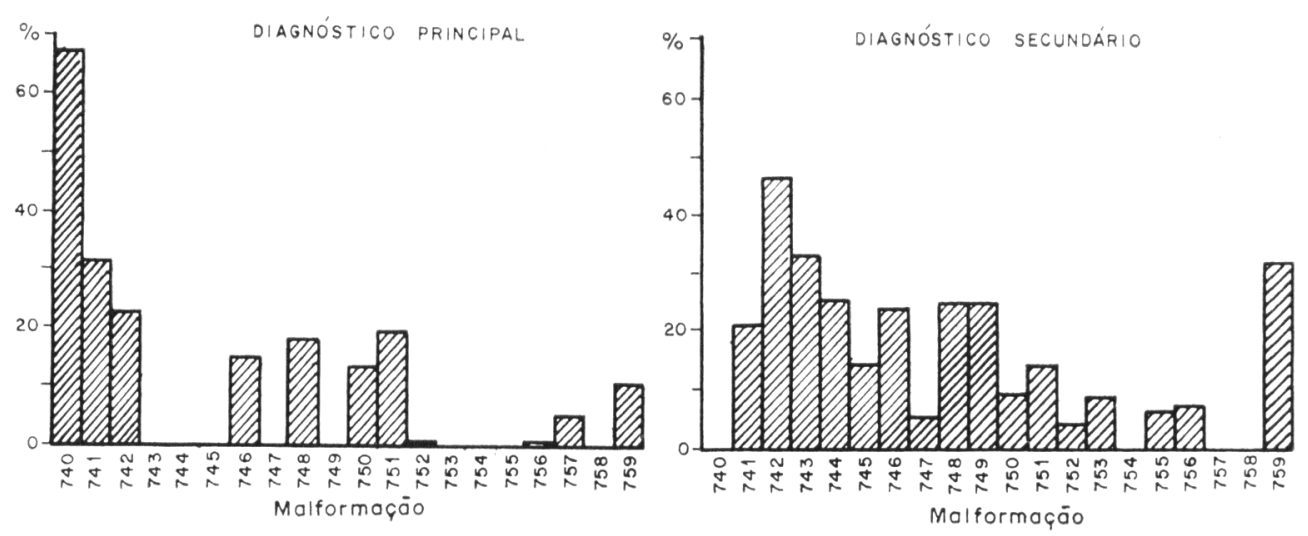

Fig. 5 - Mortalidade por cem nos diagnósticos principal e secundário de malformações. CPDH - 01/01/1970 - 30/06/1974. 
PAZ, J. E. et al. As malformações congennitas nas internações dos hospitais de Ribeirão Preto, SP, Brasil. Rev. Saúde públ., S. Paulo, 12:356-66, 1978.

Razão de sexos nas internações com diagnóstico principal de malformaçôes congênitas

A razão de sexos no total das internações com diagnóstico principal de malformaçōes congênitas difere significativamente do teórico $1: 1 \quad\left(\mathrm{X}_{1}^{2}=66,22 ; \mathrm{p}<0,001\right)$ observando-se um excesso de individuos do sexo masculino (Tabela 2). Isto está de acordo com o observado em outros estudos '. Analisando as razões de sexo por tipo de malformações, observa-se significada tendência para a sexo masculino os seguintes diagnósticos: anomalias dos olhos (744) $\left(\mathrm{X}_{1}{ }_{1}=6,78 ; \mathrm{p}<0,01\right)$; lábio leporino $\mathrm{e} / \mathrm{ou}$ palato fendido $(749) \quad\left(\mathrm{X}^{2}{ }_{1}=13,51\right.$; $\mathrm{p}<0,001)$; anomalias dos órgãos genitais (752) $\left(\mathrm{X}^{2}{ }_{1}=130,33 ; \mathrm{p}<0,001\right) ;$ pé torto
(754) $\left(\mathrm{X}_{1}{ }_{1}=27,58 ; \mathrm{p}<0,001\right)$ e outras anomalias congênitas do sistema ósteomuscular $(756)\left(\mathrm{X}^{2}{ }_{1}=5,79 ; \mathrm{p}<0,02\right)$. Em dois diagnósticos existe aumento significativo de mulheres: anomalias dos membros excluindo pé torto (755) $\left(\mathrm{X}_{1}:=6,59\right.$; $p<0,02)$ e anomalias de pele e fâneros (757) $\left(\mathrm{X}_{1}{ }_{1}=12,96 ; \mathrm{p}<0,001\right)$.

Com respeito ao lábio leporino e/ou palato fendido, por ser uma malformação muito óbvia e de definição muito precisa, comparou-se a razão de sexos observada com a razão de sexos ao nascimento, calculada na América Latina como sendo de $1,18^{2}$, e ambas não diferem significativamente $\left(X_{2}{ }_{1}=1,54 ; 0,25>p>0,20\right)$. Ao contrário, as anomalias de pele e fâneros, segundo Castilla e col.2 possuem ao nasci-

T A B E L A 3

Procedência, em percentagem, das internaçōes por malformações congènitas, por tipo de malformação - CPDH: 01/01/1970 a 30/06/1974.

\begin{tabular}{|c|c|c|c|}
\hline Diagnóstico & $\begin{array}{l}\text { Ribeirão Preto } \\
\text { Zona Urbana }\end{array}$ & $\begin{array}{c}\text { Região de } \\
\text { Ribeirão Preto } \\
\text { (Exc. Z. Urbana) }\end{array}$ & $\begin{array}{c}\text { Outras Regiões } \\
\text { do Brasil }\end{array}$ \\
\hline 740 & 33,3 & 0 & 66.7 \\
\hline 741 & 17,1 & 56.1 & 26,8 \\
\hline 742 & 22,2 & 33.8 & 44.5 \\
\hline 743 & 21,6 & 51,4 & 27,0 \\
\hline 744 & 44,1 & 32,2 & 23,7 \\
\hline 745 & 73,3 & 22.2 & 4,5 \\
\hline 746 & 38.4 & 40,9 & 20,7 \\
\hline 747 & 44,6 & 39,3 & 16,1 \\
\hline 748 & 35,3 & 35,3 & 29,4 \\
\hline 749 & 17,7 & 47,8 & 34,5 \\
\hline 750 & 43,2 & 29,7 & 27,1 \\
\hline 751 & 26,2 & 60.7 & 13,1 \\
\hline 752 & 57,1 & 33.1 & 9,8 \\
\hline 753 & 46,5 & 25,6 & 27,9 \\
\hline 754 & 36,2 & 42.7 & 21,1 \\
\hline 755 & 43,3 & 38,6 & 18,1 \\
\hline 756 & 44,1 & 36.7 & 19,2 \\
\hline 757 & 48.0 & 40.0 & 12,0 \\
\hline 758 & 66.7 & 29.2 & 4,1 \\
\hline 759 & 42,1 & 35.1 & 22,8 \\
\hline Total & 40,4 & 39.3 & 20,3 \\
\hline
\end{tabular}




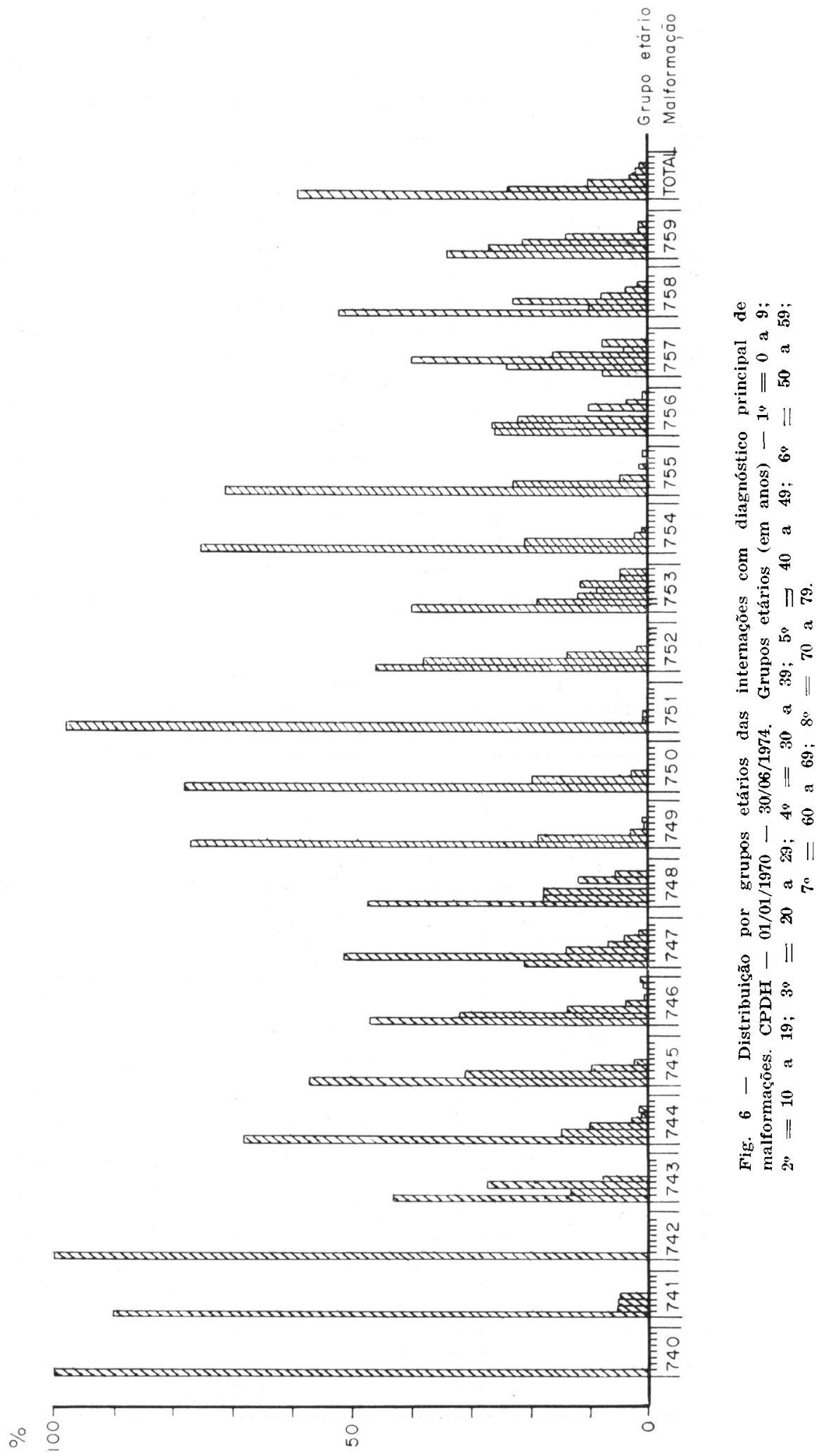


PAZ, J. E. et al. As malformações congènitas nas internações dos hospitais de Ribeirão Preto, SP, Brasil. Rev. Saúde públ., S. Paulo, 12:356-66, 1978.

mento uma razão de sexos de 1,10 ; a encontrada de 0,14 difere dela significativamente $\left(\mathrm{X}_{1}{ }_{1}=12,44 ; \mathrm{p}<0,001\right)$. Se bem que neste caso pode contribuir para essa razão de sexos anômala a preocupação maior pelo aspecto estético do sexo feminino; a demanda de assistência e correção destas malformações congênitas é mais frequentemente solicitada para tal sexo, já a razão de sexos das anomalias dos membros, excluindo o pé torto, pode-se atribuir à participação da luxação congênita do quadril, que é seis vezes mais freqüente no sexo feminino que no masculino 1 .

Duração média das internações com diagnóstico principal de malformações congênitas

A Tabela 2 mostra a duração média das internaçōes com diagnóstico principal de malformações congênitas, que foi de 15,2 dias para o total, cifra que é superior à de 12,7 dias que é a média calculada para o total do $\mathrm{CPDH}$, incluindo os Hospitais Psiquiátricos.

A duração média das internações por malformaçōes congênitas não é semelhante para todos os estabelecimentos. Comparando aqueles cinco com maior número, verifica-se que o Hospital das Clínicas possui a média maior, com 19,8 dias, seguido pela Santa Casa de Misericórdia, com 15,9 dias, o Instituto Santa Lydia, com 15,3 dias, Hospital São Francisco com 5,9 dias e o Hospital São Lucas com 4,5 dias.

E evidente o alto custo económico que representa a maior duraçāo das internações por malformaçōes congênitas, e assim as açōes para sua prevenção, tais como o aconselhamento genético, constituem-se em ações de interesse da saúde pública.
Cirurgia nas internaçóes com diagnóstico principal de malformaçôes congênitas:

Em $71,8 \%$ das internações com diagnóstico principal de malformações congênitas registradas, existe a realização de algum procedimento cirúrgico (Tabela 2 ). A maioria delas foram procedimentos destinados à correção da anomalia.

Procedência dos pacientes internados com diagnóstico principal de malformações congênitas

A Fig. 6 mostra a distribuição por idades das internações com diagnóstico principal de malformações congênitas. A média de idade para o total é de 11,6 anos, com um desvio padrão de 11,1 anos, sendo a mediana de 8,5 anos. Como pode observar-se a maioria destas distribuições são muito assimétricas com a moda no grupo etário de 0-9 anos, com exceção das malformações do aparelho circulatório (747), outras anomalias do sistema ósteo-muscular (756) e anomalias de pele e fâneros (757).

Procedencia dos pacientes internados com diagnóstico principal de malformaf̧óes congênitas

Procedem da cidade e da região de Ribeirão Preto $79,7 \%$ dos pacientes internados com diagnóstico principal de malformações congênitas (Tabela 3). O CPDH registra, para as internações nos hospitais cujos dados foram utilizados neste trabalho, sem exclusão das causas acidentais, $92,6 \%$ de tal procedência. Nota-se a diferença entre ambas quantidades $(p<0,001)$ sugerindo que os hospitais de Ribeirão Preto podem constituir-se num centro de referência para o tratamento e a correção das malformações congênitas. 
PAZ, J. E. et al. As malformações congênitas nas internações dos hospitais de Ribeirão Preto, SP, Brasil. Rev. Saúde públ,. S. Paulo, 12:356-66, 1978.

RSPUB9/424

PAZ, J. E. et al. [Congenital malformations in hospital admissions in Ribeirão Preto, State of S. Paulo, Brazil] Rev. Saúde públ., S. Paulo, 12: $356-66,1978$.

ABSTRACT: Data on admissions due to congenital malformations recorded by the Social Medicine Department Processing Center of Hospital Data, Ribeirão Preto School of Medicine, USP, registered by seven Hospitals between January $1^{\text {st }} 1970$ and July $30^{\text {th }} 1974$, are presented. These admissions represent $1.48 \%$ of the total of admissions, excluding accidents. The contribution of each hospital to these admissions is described. The distributions of the main and secondary diagnosis classified by the type of malformation were compared and showed a significant difference. A significant increase of percentages of admissions with diagnosis of congenital malformation was found throughout the years, this trend being only due to the Hospital das Clinicas. The mortality percentage, the sex ratio and the mean time of admission were also analyzed, and a significant increase was found for the latest. The analysis of surgical procedures, age distribution and patients' place of residence suggest that the hospitals in Ribeiräo Preto are serving as reference centers for treatment and correction of congenital malformations.

UnITERms: Abnormalities, Ribeirão Preto, $S P$, Brazil.

\section{REFERENCIAS BIBLIOGRAFICAS}

1. CARTER, C. O. \& WILKINSON, J. A. Genetics and environmental factors in the ethiology of the congenital dislocation of the hip. Clin. Orthop., 33: $119-28,1964$.

2. CAStilla, E. et al. Estudio latinoamericano sobre malformaciones congénitas. Bol. Ofic. sanit, panamer., $76: 494-502$. 1974.

3. FÁVERO, M. et al. Organização de um centro de informática hospitalar em nivel local. Rev. paul. Hosp., 21: 151-7, 1973.

4. MaKEOWN, T. \& RECORD, R. Malformations in a population observed for five sears after birth, In: Cibat Symposium on Congenital Malformations. London, J. \& A. Churchill. 1960. p. 2-21.

5. ORGANIZAÇAO PANAMERICANA DA SAƯDE. Manual da classificação estatística internacional de doenças, lesões e causas de bbito; oitava revisão: 1965 Washington. D. C. 1969. (Publ. cient. 190).

6. SMITHELLS, R. W. The early diagnosis of congenital abnormalities. London, Cassell. 1963.

Recebido para publicaçāo em 31/01/1978 Aprovado para publicaşa em 13/04/1978 